\title{
ASTRONOMY ON THE MIDCOURSE SPACE EXPERIMENT
}

\author{
S.D. PRICE ${ }^{1}$, E.F. TEDESCO ${ }^{2}$, M. COHEN ${ }^{3}$, \\ R.G. WALKER ${ }^{4}$, R.C. HENRY ${ }^{5}$, M. MOSHIR ${ }^{6}$, \\ L.J. PAXTON ${ }^{7}$ AND F.C. WITTEBORN ${ }^{8}$ \\ ${ }^{1}$ Phillips Laboratory, Hanscom AFB, \\ ${ }^{2}$ Mission Research Corporation, \\ ${ }^{3}$ Univ. of California Radioastronomy Laboratory, \\ ${ }^{4}$ Jamieson Science $\mathscr{G}$ Engineering, Inc., \\ ${ }^{5}$ Johns Hopkins University, \\ ${ }^{6}$ Jet Propulsion Laboratory, \\ ${ }^{7}$ Applied Physics Laboratory, \\ ${ }^{8}$ NASA/Ames Research Center
}

\begin{abstract}
The Midcourse Space Experiment (MSX) carries a varied suite of sensors for imaging in the ultraviolet to the mid-infrared, hyperspectral imaging in the ultraviolet through visible and infrared spectroscopy with a Michelson interferometer. At comparable sensitivity to the Infrared Astronomical Satellite (IRAS) but with a 40 times smaller point response function, the MSX infrared radiometer is ideal for surveying specific large areas such as those in which IRAS was degraded by confusion or not covered at all. This experiment obtains simultaneous observations over a very wide spectral range, from 0.11 to $25 \mu \mathrm{m}$, thus providing unique information on the energetics of such diverse objects as comets and H II regions. Initial observations indicate that the astronomy objectives on this experiment will be achieved.
\end{abstract}

\section{Introduction}

The Midcourse Space Experiment (MSX) is a Ballistic Missile Defense Organization mission for research on space-based tracking of Ballistic Missiles. This includes a variety of measurements related to the chemistry and physics of the atmosphere and astronomy. MSX was successfully launched on 24 April 1996 into a $\sim 900 \mathrm{~km}$ altitude circular orbit. The four to five 
year mission lifetime is divided into a cryogen phase lasting about a year during which infrared measurements are a priority and the post cryogen phase using the suite of ultraviolet through visible (red) sensors. The infrared astronomy survey objectives are to cover the regions either missed by previous experiments such as the IRAS gaps and the zodiacal cloud near the sun and in the anti-solar direction or where previous experiments were degraded by confusion such as in the Galactic Plane. Additional measurements probe Galactic structure, the physical processes in $\mathrm{H}$ II regions and comets, obtain spectroscopy on extended sources and tie a set of secondary stellar calibration standards to the generally adopted primary stars. The post cryogen astronomy goal is to survey the sky in the "solar-blind" UV.

\section{Instrumentation}

An overview of the spacecraft, instruments and large range of scientific objectives of this mission is given by Mill et al. (1994) with more detail provided in Vol. 17, Numbers 1 and 2 of the Johns Hopkins APL Technical Digest (1996). The instruments used for astronomy on MSX are the SPatial InfraRed Imaging Telescope (SPIRIT) III, the four large and small field ultraviolet and visible imaging instruments (Imagers) and the five hyperspectral uv-visible imagers (SPIMs).

SPIRIT III is a $35 \mathrm{~cm}$ diameter clear aperture off-axis telescope with two focal plane instruments, five line scanned arrays and a Michelson interferometer. The spectral responses of the line scanned arrays are 6 to 11 $\mu \mathrm{m}$ (designated Band A), 11-13 $\mu \mathrm{m}$ (Band C), 13-15 $\mu \mathrm{m}$ (Band D), 18 to $25 \mu \mathrm{m}$ (Band E) and a very narrow $(\sim 0.25 \mu \mathrm{m})$ region at $4.2 \mu \mathrm{m}$ (Band B). Each array is 8 columns by 192 rows of $18^{\prime \prime}$ pixels with half the columns offset by half a pixel. Only half the columns are active to reduce the data rate to only $5 \mathrm{Mbps}$. The interferometer is capable of spectral resolutions from 2 to $20 \mathrm{~cm}^{-1}$. Three large detectors, $12^{\prime} \times 12^{\prime}$, divide up the spectral region between 6 and $28 \mu \mathrm{m}$, two of the $6^{\prime}$ by $9^{\prime}$ elements cover the entire 2.7 to $28 \mu \mathrm{m}$ range while the third is limited to 2.7 to $5 \mu \mathrm{m}$.

The narrow field visible and ultraviolet instruments image $1.3^{\circ} \times 1.6^{\circ}$ fields with pixels slightly larger than those in SPIRIT III. The wide field imagers have eight times larger pixel with the corresponding increase in field size. Collectively, the imagers span the spectral range from 110 to $900 \mathrm{~nm}$. Five hyperspectral imagers combined cover 110 to $893 \mathrm{~nm}$ at a resolving power as high as 200 . Uniquely, the SPIMs can provide spectra in $1.5^{\prime}$ increments over a linear spatial extent of $1^{\circ}$ or over a $1^{\circ}$ by $1^{\circ}$ field. 


\section{Astronomy Experiments on MSX}

With Band A having an on orbit, single read sensitivity comparable to the IRAS $12 \mu \mathrm{m}$ band and a footprint some 40 times smaller, a principal experiment is to survey the Galactic Plane, the Magellanic Clouds and star forming regions in the large molecular clouds in which IRAS was confused. The area within $5^{\circ}$ of the Plane will be redundantly covered once and within $3^{\circ}$ twice. Deeper probing of Galactic structure, by at least a factor of 20 in sensitivity, is obtained from raster scans of selected regions in the plane. Another priority is to survey areas not previously covered by other infrared experiments. This includes the IRAS gaps and, owing to the superior side-lobe rejection of SPIRIT III, solar elongations between $25^{\circ}$ and $30^{\circ}$ and greater than $125^{\circ}$. Other infrared experiments include observations of several large galaxies, comets and asteroids and infrared secondary calibration standards. The interferometer obtains spectroscopy on the emission characteristics of the zodiacal dust cloud and the Galactic Plane during the survey scans. Spectroscopy of extended sources will be emphasized during the last half year of the cryogen phase of the mission.

The wide field UV imager has at least ten times smaller pixels and higher sensitivity than TD-1. Thus, the primary post cryogen objective is to survey the sky with this instrument while sampling in the higher resolution and sensitivity narrow field UV imager and SPIMs. The visible imagers will concurrently survey for small, relatively near, low activity comets by looking for $\mathrm{OH}^{-}$emission from these objects.

\section{Acknowledgements}

We are grateful for the work of our Associate Investigators, M. Allen, M. Egan, J. Murthy, R. Shipman, and J. Simpson.

\section{References}

Johns Hopkins APL Technical Digest, 1994, Vol. 17, No. 1, 2-116 and No. 2, 134-252. Mill, J.D., O'Neil, R.R., Price, S., Romick, G.J., Uy, O.M., Gaposchkin, E.M., Light, G.C., Moore, Jr., W.W., Murdock, T.L., \& Stair, A.T., 1994, "Midcourse Space Experiment: Introduction to the Spacecraft, Instruments, and Scientific Objectives, Jour. Spacecraft and Rockets, Vol. 31, No. 5. 900-907. 